\title{
Azimuthal asymmetries in production of charged hadrons by high energy muons scattered off polarised deuterons at COMPASS.
}

\author{
Igor A. Savin ${ }^{1}$, on behalf of the COMPASS Collaboration \\ Joint Institute for Nuclear Research \\ Dubna, Russia \\ E-mail: Igor.Savin@cern.ch
}

\begin{abstract}
Azimuthal asymmetries in semi-inclusive production of charged hadrons by muons scattered off longitudinally polarised deuterons have been searched for in the COMPASS experiment at CERN. The asymmetries are parameterised taking into account possible contributions from different nucleon parton distribution functions depending on the transverse or longitudinal components of the quark spin and parton fragmentation functions. They can be modulated with $\sin (\phi), \sin (2 \phi), \sin (3 \phi)$ and $\cos (\phi)$. The parameterisation includes also a $\phi$ - independent term.

The amplitudes of all $\phi$-modulation terms for hadrons integrated over kinematic variables are found to be consistent with zero, while the $\phi$-independent terms are non zero and about equal for positive and negative hadrons.

The dependence of the parameterization parameters on the kinematic variables $\mathrm{x}, z$ and $p_{T}^{h}$ is also studied. The $\mathrm{x}$-dependence of the $\phi$ - independent term is found to be in agreement with the COMPASS data on the hadron asymmetry $A_{d}^{h}(x)$.The x-dependence of the sin $(\phi)$ modulation amplitude is observed while dependence of other modulation amplitudes is consistent with no variation vs. the kinematic variables.
\end{abstract}

XVIII International Workshop on Deep-Inelastic Scattering and Related Subjects - DIS 2010

Firenze, Italy

April 19-23 2010

1 Speaker 


\section{Introduction}

The quark transverse momentum dependent distributions in nucleons, leading to an appearance of azimuthal asymmetries (AA) of hadrons produced in Semi-Inclusive Deep Inelastic Scattering (SIDIS), have been predicted theoretically (see [1] and references therein) and seen for the first time by the SMC, HERMES and CLAS [2] experiments. These asymmetries result from a convolution of Parton Distribution Functions (PDF) depending on the transverse or longitudinal component of the quark spin and Parton Fragmentation Functions (PFF). The AAs on the transversely polarised protons and deuterons have been studied by HERMES [3] and COMPASS [4] Collaborations, and on the longitudinally polarised protons and deuterons - by HERMES [5], [6]. The AAs on the deuterons are found to be much smaller than that on protons indicating a different signs of contributions from u- and d-quarks. The search for the AA using the COMPASS spectrometer [7] with the longitudinally polarised deuterons in a wide kinematical range is presented here.

The kinematics of the SIDIS is shown in Fig.1a with a usual signature of variables in the muon scattering and hadron production planes.

In the framework of the parton model of nucleons, the squared modulus of the matrix element of the SIDIS is represented by the diagram of the type shown in Fig.1b. The spin dependent transversity PDF, $h_{1}(x)$, and the Collins PFF, $H_{1}^{\perp}(z)$, are given as examples. More PDFs enter in the total SIDIS cross section. In general, this cross section is a linear function of the muon beam polarisation, $P_{\mu}$, and of the target polarization components:

$$
d \sigma=d \sigma_{00}+P_{\mu} d \sigma_{L 0}+P_{L}\left(d \sigma_{0 L}+P_{\mu} d \sigma_{L L}\right)+\left|P_{T}\right|\left(d \sigma_{0 T}+P_{\mu} d \sigma_{L T}\right),
$$

where the first (second) subscript of the partial cross section refers to the beam (target) polarisation. The asymmetry, a $(\phi)$, in the hadron production by polarised muons on the target longitudinally polarised (LPT) along or opposite to the muon beam direction, is proportional to the third and fourth terms in Eq. (1) if the beam polarisation remains unchanged:

$$
a(\phi)=\frac{d \sigma^{\leftarrow \Rightarrow}-d \sigma^{\leftarrow \leftarrow}}{\left|P_{L}\right|\left(d \sigma^{\leftarrow}+d \sigma^{\leftarrow}\right)} \sim-\left(d \sigma_{0 L}+P_{\mu} d \sigma_{L L}\right)+\tan \left(\theta_{\gamma}\right)\left(d \sigma_{0 T}+P_{\mu} d \sigma_{L T}\right) .
$$

Each partial cross section in Eq. (2) is characterized by terms including a convolution of PDFs and PFFs multiplied by a function of the hadron azimuthal angle.

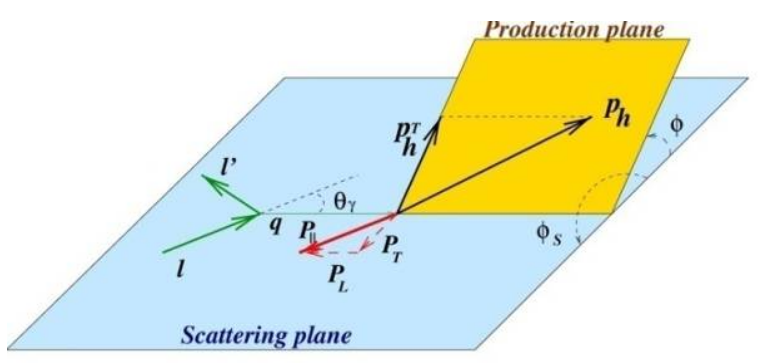

(a)

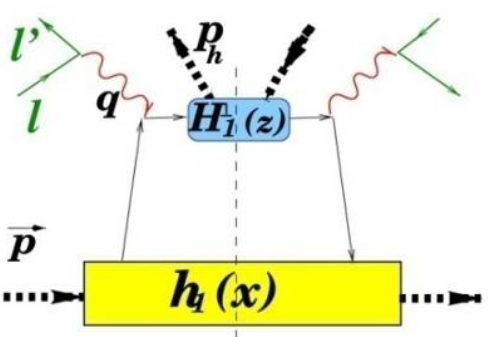

(b)

Fig.1: (a) The kinematics of the process. (b) Squared modulus of the matrix element of the SIDIS reaction $\ell+\vec{N} \rightarrow \ell^{\prime}+h+X$ summed over $\mathrm{X}$ states.

Namely, contributions to Eq. (2) from each quark and antiquark flavor, up to the order $M / Q$, have the forms: 


$$
\begin{aligned}
& d \sigma_{0 L} \propto x h_{1 L}^{\perp}(x) \otimes H_{1}^{\perp}(z) \sin (2 \phi)+\frac{M}{Q} x^{2}\left[h_{L}(x) \otimes H_{1}^{\perp}(z)+f_{L}^{\perp}(x) \otimes D_{1}(z)\right] \sin (\phi), \\
& d \sigma_{L L} \propto x g_{1 L}(x) \otimes D_{1}(z)+\frac{M}{Q} x^{2}\left[g_{L}^{\perp}(x) \otimes D_{1}(z)+e_{L}(x) \otimes H_{1}^{\perp}(z)\right] \cos (\phi), \\
& d \sigma_{0 T} \propto x h_{1}(x) \otimes H_{1}^{\perp}(z) \sin \left(\phi+\phi_{S}\right)+x h_{1 T}^{\perp}(x) \otimes H_{1}^{\perp}(z) \sin \left(3 \phi-\phi_{S}\right) \\
& \quad-x f_{1 T}^{\perp}(x) \otimes D_{1}(z) \sin \left(\phi-\phi_{S}\right), \\
& d \sigma_{L T} \propto x g_{1 T}(x) \otimes D_{1}(z) \cos \left(\phi-\phi_{S}\right) .
\end{aligned}
$$

The symbol $\otimes$ represents convolutions in the intrinsic parton transverse momentum on which PDFs and PFFs depend. The structure of the partial cross sections and physics interpretations of the PDFs and PFFs in a $(\phi)$ are given in [1].

So, the aim of this study is to see the AA in the hadron production from LPT, as a signal of quark spin dependent PDFs and PFFs, responsible for sin $(\phi)$, sin $(2 \phi)$, sin $(3 \phi)$ and $\cos (\phi)$ modulations, and to test the $\mathrm{x}, \mathrm{z}$ and $p_{T}^{h}$ dependence of the corresponding amplitudes.

\section{Method of analysis and data selection}

The experiment is performed in the muon beam M2 at CERN with the COMPASS spectrometer described elsewhere [7]. The muons of the beam originate mainly from the pion and kaon two body decays. They are naturally polarised with the average $P_{\mu}=-80 \%$.

The method of analysis takes advantage of the COMPASS polarised target and data taking procedure. In the years 2002-2004 the target consisted of two cells labeled "U" and "D" ( Upand Down-stream of the setup) placed in the $2.5 \mathrm{~T}$ solenoid magnet centered along the beam direction. The target material of the cells $\left({ }^{6} \mathrm{LiD}\right)$ was polarised in opposite directions (positive and negative with respect to the beam) using the Dynamic Nuclear Polarisation. Such a configuration is achieved at a certain orientation (positive or negative) of the solenoid field.

The data are taken simultaneously from two cells. There are two sources of systematic effects connected with collection of data, first, due to possible time variation of the spectrometer acceptance, second, due to possible dependence of the acceptance on the orientation of the solenoid field. The first source has been minimised by frequent (three times a day) changing of the cell polarisations adiabatically reversing the solenoid field and, second, - by realising (after few weeks of data taking) the initial configuration of the cell polarisations with the opposite orientation of the solenoid field exchanging the microwave frequency of the cells.

For the AA studies the double ratios of event numbers, $\mathrm{R}_{\mathrm{f}}$, is used in the following form:

$$
R_{f}(\phi)=\left[N_{+, f}^{U}(\phi) / N_{-, f}^{D}(\phi)\right] \cdot\left[N_{+, f}^{D}(\phi) / N_{-, f}^{U}(\phi)\right] .
$$

Here $N_{p, f}^{t}(\phi)$ is a number of events in each $\phi$-bin from the target cell t, t=U, D, p=+ or - is the sign of the target polarisation, $\mathrm{f}=+$ or - is the direction of the target solenoid field. The ratio depends only on physics characteristics of the SIDIS process. It can be expressed via asymmetry a $(\phi)$, Eq. (2), in the quadratic equation, approximate solution of which is:

$$
a_{f}=\left[R_{f}(\phi)-1\right] /\left(P_{+, f}^{U}+P_{+, f}^{D}+P_{-, f}^{U}+P_{-, f}^{D}\right) .
$$

The $P_{p, f}^{t}$ is an absolute value of averaged products of the target cell polarisation and dilution factor. Since the asymmetry should not depend on the direction of the solenoid field, one can 
expect to have $\mathrm{a}_{+}=\mathrm{a}$. This expectation has been checked with the data. So, the weighted sum a $(\phi)=a_{+}(\phi)+a_{-}(\phi)$, calculated separately for each year of data taking and averaged at the end, is obtained for the final results.

The data selection, aimed at having a set of clean hadrons, has been performed starting from a preselected sample of SIDIS events with $\mathrm{Q}^{2}>1(\mathrm{GeV} / \mathrm{c})^{2}$ and $\mathrm{y}>0.1$, the $\mathrm{Q}^{2}$ range of which extends up to $100(\mathrm{GeV} / \mathrm{c})^{2}$. The sample contains all data collected in 2002-2004. Each event includes a reconstructed vertex with incoming and outgoing muons and one or more additional outgoing tracks. Applying cuts on the quality of reconstructed tracks and vertices, vertex positions inside the target cells, energy transfer $(y<0.9)$ and invariant mass of the final states $(5<\mathrm{W}<18 \mathrm{GeV})$, about $96 \mathrm{M}$ of SIDIS events have been selected for further analysis in the incoming muon momentum range $140-180 \mathrm{GeV} / \mathrm{c}$.

Tracks originating from the SIDIS events have been identified as hadrons using the information from the two hadron calorimeters available at COMPASS. The total number of the hadrons is about $53 \mathrm{M}$. Each hadron enters the asymmetry evaluation which has been done in the restricted kinematic region of $\mathrm{x}=0.004-0.7, \mathrm{z}=0.2-0.9, p_{T}^{h}=0.1-1 \mathrm{GeV} / \mathrm{c}$.

\section{Results}

The weighted sums of the azimuthal asymmetry a $(\phi)=a_{+}(\phi)+a_{-}(\phi)$ for negative and positive hadrons have been fitted by a function:

$$
a(\phi)=a^{\text {const }}+a^{\sin \phi} \sin (\phi)+a^{\sin 2 \phi} \sin (2 \phi)+a^{\sin 3 \phi} \sin (3 \phi)+a^{\cos \phi} \cos (\phi),
$$

where the fit parameters can depend on the variables $\mathrm{x}, \mathrm{z}$ and $p_{T}^{h}$. The asymmetries, first, have been calculated for hadrons integrated over all kinematic variables, and, second, - as a function of one of variables for hadrons integrated over other two.

For hadrons integrated over kinematic variables, the parameters, characterising $\phi$-modulation amplitudes, are compatible with zero while the $\mathrm{a}^{\text {const }}$ differ from zero and equal to $(2.7+-1.1)$ ppm and (3.0+-1.1) ppm for negative and positive hadrons, respectively. As already specified, the $\mathrm{a}^{\text {const }}$ terms come from the $\mathrm{d} \sigma_{\mathrm{LL}}$ contribution to the asymmetry, which is proportional to the helicity PDF $g_{1}$ convoluted with the PFF $D_{l}$ (see Eq. (3)) of non-polarised quarks in a nonpolarised hadron. For the deuteron target these terms are expected to be charge independent, as

it is confirmed by the above numbers. They are related to the hadron asymmetries $A_{d}^{h}$ for the negative and positive hadrons.

Dependence of the AA fit parameters on the kinematic variables is shown in Fig. 2 with the statistical errors only. Systematic errors are estimated to be much smaller.

The parameters $\mathrm{a}^{\text {const }}(\mathrm{x})$, being divided by a product of the average muon polarisation and virtual photon depolarisation factor in corresponding $\mathrm{x}$ bin, $\mathrm{D}_{0}$, by definition, are equal to the asymmetries $A_{d}^{h}$, published by the COMPASS [8]. Agreement of these data with those of the present analysis has demonstrated the internal consistency of the COMPASS results.

The amplitudes $a^{\sin \phi}$ are related to the twist-3 PDFs $h_{L}$ and $f_{L}$ entering $\mathrm{d} \sigma_{0 \mathrm{~L}}$ as well as to the twist- 2 transversity PDF $h_{1}$ and Collins PDF $f_{I T}$ entering $d \sigma_{0 T}$. All of them contribute to the AA with a factor $\sim \mathrm{Mx} / \mathrm{Q}$. The observed $\mathrm{x}$ dependence of this modulation is less pronounced in the COMPASS than in the HERMES data [6]. One can note also that the last ones are obtained for leading pions, while our data include all SIDIS hadrons and cover a much wider range in $\mathrm{x}, \mathrm{Q}^{2}$ and W. Restricting our kinematic region to that of the HERMES for the amplitude $a^{\sin \phi}$ we obtain compatible results.

The amplitudes of the sin $(2 \phi)$ modulation are small, consistent with zero within the errors. They could be caused by PDF $h_{1 L}^{\perp}$ in $\mathrm{d} \sigma_{0 \mathrm{~L}}$ which is approximately linked [9] with the 
transversity PDF $h_{1}$ by a relation of the Wandzura- Wilczek type. Predictions [9] are shown by dashed (solid) line for $\mathrm{h}-(\mathrm{h}+)$, respectively.

The amplitudes $a^{\sin 3 \phi}$ are compatible with zero as the COMPASS results on the amplitude of the $\sin \left(3 \phi-\phi_{S}\right)$ modulation extracted from a transversely polarised target [10] ( $\phi_{S}=0$ for the LPT). This modulation would be due to the pretzelosity PDF $h_{1 T}^{\perp}$ in $\mathrm{d} \sigma_{0 \mathrm{~T}}$, suppressed by a factor $\sim \mathrm{xM} / \mathrm{Q}$.

The cos $(\phi)$ modulation of the AA from the LPT is studied here for the first time. The data are consistent with no variations of the modulation amplitudes vs. kinematical variables. This modulation is proportional to the muon beam polarisation and would be due to a pure twist-3 PDF $g_{L} \frac{1}{L}$ in $d \sigma_{\mathrm{LL}}$, an analog to the Cahn effect [11] in unpolarised SIDIS [12], and $g_{I T}$ in $d \sigma_{L T}$ suppressed by a factor $\sim \mathrm{xM} / \mathrm{Q}$.
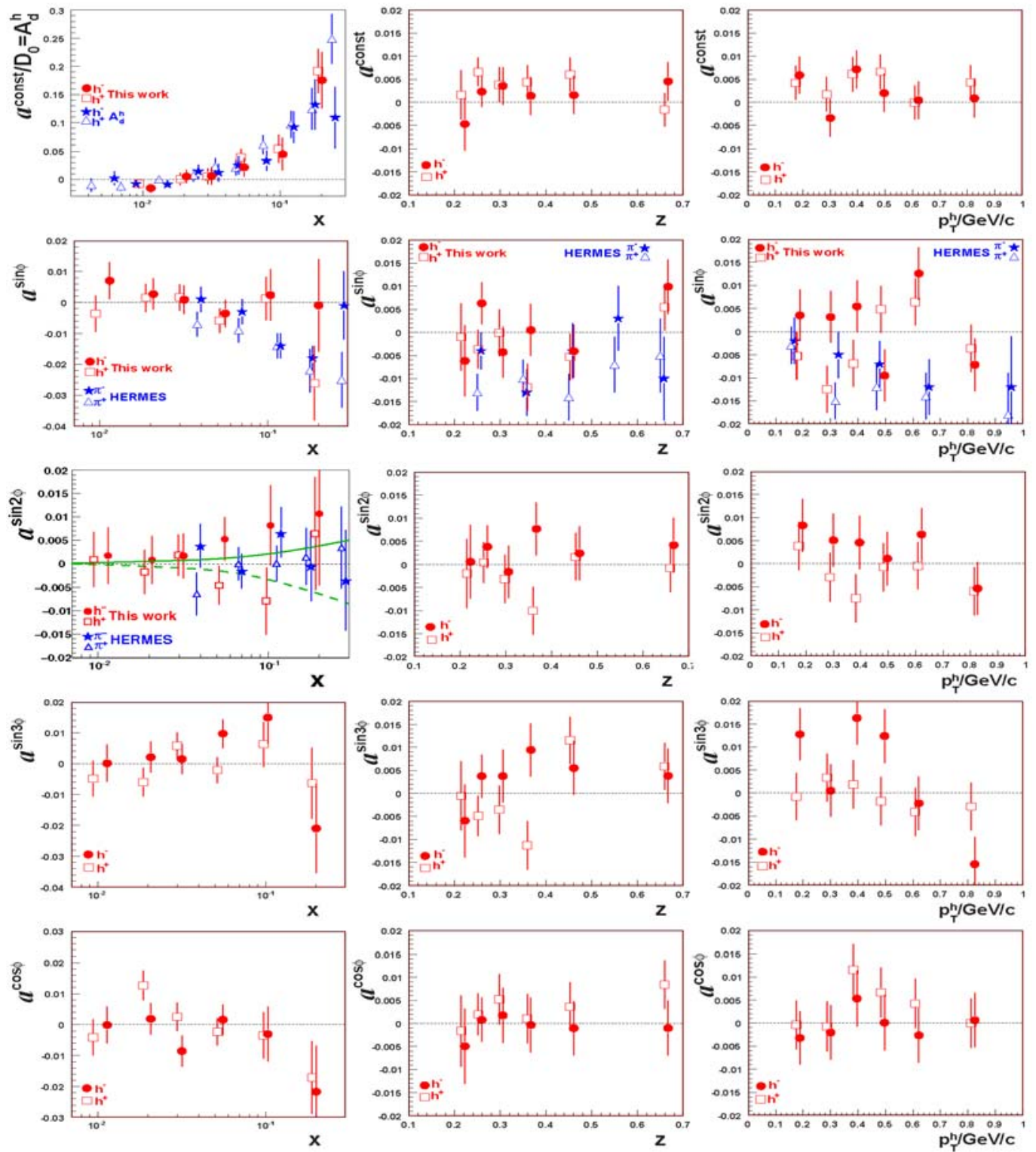

Fig.2. Dependence of the AA fit parameters on the kinematic variables. 


\section{Conclusions}

The azimithal asymmetries in the SIDIS production of negative and positive hadrons by 160 $\mathrm{GeV}$ muons on the longitudinally polarised deuterons have been studied. For hadrons integrated over $\mathrm{x}, \mathrm{z}$ and $p_{T}^{h}$ all $\phi$ - modulation amplitudes of the $\mathrm{a}(\phi)$ are consistent with zero, while the $\phi$ independent parts differ from zero and are equal for $\mathrm{h}^{-}$and $\mathrm{h}^{+}$within the statistical errors.

The modulation amplitudes as functions of the kinematic variables are studied. The $\phi$ independent parts of the a $(\phi), a^{\text {oonst }}(x) / D_{0} \equiv A_{d}^{h}(x)$ are in agreement with the COMPASS published data [8] on $A_{d}^{h}(x)$. The amplitudes $a^{\sin \phi}\left(x, z, p_{T}^{h}\right)$ are small and compatible with the HERMES data [6], if one takes into account the difference in $\mathrm{x}, \mathrm{Q}^{2}$ and $\mathrm{W}$ between the two experiments. The amplitudes $\mathrm{a}^{\sin 2 \phi}\left(\mathrm{x}, \mathrm{z}, p_{T}^{h}\right), a^{\sin 3 \phi}\left(x, z, p_{T}^{h}\right)$ and $a^{\cos \phi}\left(x, z, p_{T}^{h}\right)$ are consistent with zero within the statistical errors.

\section{References.}

[1] A.Bacchetta et al., JHEP 0702 (2007) 093.

[2] SMC, A. Bravar, Nucl.Phys.Proc.Suppl. 7 (1999) 52; HERMES, A. Airapetian et al., Phys.Rev.Lett. 87 (2001) 182001 ;

CLAS, S. Stepanyan et al., Phys.Rev.Lett 87 (2001) 182002.

[3] HERMES, A. Airapetian et al., Phys.Rev.Let. 94 (2005) 012002.

[4] COMPASS, E.S. Ageev et al., NP B 765 (2007) 31.

[5] HERMES, A .Airapetian et al., Phys.Rev.Lett. 84 (2000) 4047; Phys.Rev. D64 (2001) 097101; Phys.Lett. B622 (2005) 14.

[6] HERMES, A. Airapetian et al., Phys.Lett. B562 (2003) 182.

[7] COMPASS, P. Abbon et al., NIM A577 (2007) 31-70.

[8] COMPASS, M. Alekseev et al., Phys.Lett. B660 (2008) 458.

[9] H. Avakian et al., Phys.Rev. D77 (2008) 014023.

[10] A. Kotzinian, on behalf of the COMPASS collaboration, [arXiv : 0705.2402 [hep-ex]].

[11] R.N. Cahn, Phys.Lett. B78 (1978) 269; Phys.Rev. D40 (1989) 3107

[12] EMC, M. Arneodo et al., Z. Phys. C 34 (1987) 277. 